\title{
Anti-inflammatory effect of plant compositions of Tibetan medicine in vitro on early rheumatoid arthritis
}

\author{
L. Yu. Semenova*, J. M. Salmasi, G. V. Poryadin
}

Department pathophysiology, The Russian State Medical University, Moscow, Russia;

*Corresponding Author: sememovaly@mail.ru

Received 27 May 2010; revised 9 June 2010; accepted 22 June 2010.

\begin{abstract}
Materials and Methods: lymphocytes of 10 patients having early rheumatoid arthritis (RA) (the duration of the illness was 3 - 6 months) with a marked exudational process in joints were examined. The content of lymphocytes expressing the CD3, CD4, CD8, CD16, CD56, CD20, CD72, CD38, CD23, CD25, CD71, HLA-DR, CD95, CD30, CD54, mlgM, mlgG antigens was determined. Results: the "Taban-Arshan" extract corrects the changes of the immune system characterized by the evident activation of the B-cell part of the immune system and normalizes immune parameters of the lymphocytes taken from the patients with autoimmune diseases (early rheumatoid arthritis). The immunocorrective effect of the "Taban-Arshan" extract is related to its ability to suppress the lymphocyte increased activation by normalizing expression of the main activation antigens (CD23, CD25, CD71, HLA-DR, CD54).
\end{abstract}

Keywords: Early Rheumatoid Arthritis;

Subpopulation of Lymphocytes; Tibetan Medicine; Immunoregulators

\section{INTRODUCTION}

The elucidation of the role of the immune system in physiological and pathophysiological processes creates prerequisites for developing disease treatment methods based on directional regulation of immune reactions. [1-4].

Current experience in application of immunoregulators in treatment of autoimmune diseases demonstrated certain success in this direction; however, some immunomodulators used in clinical medicine nowadays have adverse actions upon the organism. Thereupon researchers' attention is drawn by phytogenous immunotropic drugs that have been widely studies over the past years.

At present phytogenous drug compositions prepared according to Tibet medicine prescriptions arouse a serious interest. It is known that herb extracts and tinctures have no adverse effects and possess apparent regulating properties. Effectiveness of Tibetan medicine treatment methods has been checked by a centuries-old practice and has raised no doubts till now [5-12].

One of the promising Tibetan medicine plant compositions is "Taban-Arshan" that is mostly helpful in disorders of the locomotor apparatus. A decoction of this phyto collection saturates the organism with oxygen, produces a favorable action on metabolism, as well as removes edemata, pains in joints and reflex muscle spasms. "Taban-Arshan" is used in a folk medicine to treat diseases of joints, osteochondrosis, kidney and urogenital diseases, female disorders and skin diseases $[12,13]$. "Polyphytokhol", a complex plant drug of the Tibetan medicine has hepatoprotective and hemostimulating effects and is used in diseases of the hepatobiliary system [14-16]. Another combined phytodrug-"Baikalsky"-produces anti-inflamatory and expectorative effects and is used in diseases of the upper respiratory tract [17].

Three herbal drug compositions of the Tibetan medicine were offered for our studies by the Ulan-Ude Center of Eastern Medicine. Since collections of herbs of the traditional Tibetan medicine have been used from old times to treat inflammatory processes, it was of interest to define more exactly if their anti-inflammatory effect is connected with their influence on the immune system.

The aim of the present research work was to study the expression of surface antigens by lymphocytes of healthy donors and sick persons under the effect of the extracts "Taban-Arshan", "Polyphytokhol" and "Baikalsky" extracts of the Tibetan medicine.

\section{MATERIALS AND METHODS}

Lymphocytes of 10 patients having early rheumatoid 
arthritis (RA) (the duration of the illness was 3-6 months) with a marked exudational process in joints were examined. The RA diagnosis was based on the American Rheumatologic Association's criteria. All patients' diagnoses were confirmed by X-ray data. At the time of the study the patients received no basis therapy. 30 practically healthy persons of 20 - 30 years old served as controls. To conduct the immunological analysis, the blood was taken from the ulnar vein and stabilized by Trilon B solution. The lymphocyte suspension was obtained in the density gradient of phycol-verographin according to Boyum's method. The content of lymphocytes expressing the CD3, CD4, CD8, CD16, CD56, CD20, CD72, CD38, CD23, CD25, CD71, HLA-DR, CD95, CD30, CD54, mIgM, mIgG antigens was determined by the method of indirect immunofluorescence using monoclonal antibodies of the IKO and LT series (Russia).

To determine the effect of the extracts on the expression of lymphocyte surface receptors, a sterile separation and cultivation of cells in the final concentration of 2.5 million/ml in a minimal medium 199 with no antibiotic in the atmosphere of $5 \% \mathrm{CO}_{2}$ at a temperature of $37^{\circ} \mathrm{C}$ for 16 hours were conducted. The concentration of the preparations in the incubation medium was $10^{-7} \mathrm{M}$.

The results were computer processed according to the method of variational statistics by the Statistika application program package; the validity of differences was estimated by Student's criteria.

\section{RESULTS AND DISCUSSION}

To evaluate the patients' immune status, population and subpopulation composition of T-lymphocytes was studied as well as quantitative composition of B-lymphocytes, content of NK-cells and parameters of the activation process in lymphocytes were determined.

The effect of the extracts on the expression of surface antigens of lymphocytes of 10 healthy donors during the cultivation process was previously analyzed.

The conducted studies have shown that the cultivation process of cells in vitro for 16 hours causes a change in the expression of some subpopulations of lymphocytes (CD3, CD8, CD16). Perhaps it is due to a loss of receptors or an increase of their expression (Table 1).

The obtained results show that under the influence of the "Taban-Arshan" extract in vitro there are neither essential nor valid changes in the subpopulation of the T-lymphocytes picked out of the healthy donors-total content of the T-lymphocytes, helper $\left(\mathrm{CD}^{+}\right)$and cytotoxic $\left(\mathrm{CD}^{+}\right)$lymphocytes. In this study it was also shown that the content of the subpopulation of natural killers (CD16) and natural killers that realize their cytotoxic function (CD56) did not differ much from the levels of the healthy persons with the "Taban-Arshan" ex- tract used.

The obtained results show that the "Polyphytokhol" extract causes an increase of the expression of the total quantity of T-lymphocytes (CD3) as well as a decrease of quantity of the natural killers realizing their cytotoxic function (CD56). The cell cultivation with the "Baikalsky" extract results in a valid increase $(p<0.05)$ of the contents the cytotoxic $\left(\mathrm{CD}^{+}\right)$lymphocytes in healthy donors and a rise of the surface receptor (CD16) expression (Table 1).

During the studies of the B-cell part of the immune system, no valid changes in B-lympocytes content (CD20 ${ }^{+}$-cells) and their subpopulation composition were registered in the cell culture picked out from the healthy donors under the influence of the "Taban-Arshan" extract (Table 2).

Under the influence of the drug compositions "Polyphytokhol" and "Baikalsky" a reduction of B-lymphocyte $\left(\mathrm{CD} 20^{+}\right)$expression was observed. Moreover, the "Baikalsky" extract changed the B-lymphocyte subpopulation composition of a various degree of differentiation making the CD38, mIgG expression lower (Table 2).

Changes of the cell culture content of various activation antigens in vitro did not differ significantly from similar values of the healthy persons with the "Taban-Arshan" extract used.

The surface phenotype of the B-lymphocytes expressing marker CD23 in the process of cultivation of lymphocytes taken from the healthy donors under influence of the "Baikalsky" extract showed a tendency to increasing CD23 quantity (Table 3). The expression of the receptor for transferrin-CD71 (that is an early activation marker) had a tendency to increase this factor when coupled with the "Baikalsky" extract. It should be noted that the quantity of lymphocytes taken from the healthy donors that expressed "the late" activation antigen HLA-DR in the presence of the "Polyphytokhol" and "Baikalsky" extracts did not correspond to the similar factor of the healthy persons during cultivation without the extracts. The quantity of lymphocytes that expressed the HLA-DR activation antigen was reduced compared with the reference level (Table 3).

A tendency to increasing the number of lymphocytes expressing the ICAM-1 (CD54) adhesion marker, which were taken from the healthy donors was registered with the use of "Baikalsky" extract. It means that there is no full stop of the stimulation process of the immune system.

The results of the investigation of the "Polyphytokhol" and "Baikalsky" extracts effects on the expression of surface markers of the lymphocytes taken from the healthy donors testify the absence of any toxic effect of the examined extracts of the used concentration. The 
Table 1. Change of the expression of surface antigens of subpopulations of T- and NK-lymphocytes taken from the healthy donors during cultivation under the influence of the "Taban-Arshan", "Polyphytokhol" and "Baikalsky" extracts (in \%).

\begin{tabular}{|c|c|c|c|c|c|}
\hline \multirow[b]{2}{*}{$\begin{array}{l}\text { Lymphocyte } \\
\text { markers }\end{array}$} & \multirow{2}{*}{$\begin{array}{l}\text { Lymphocytes of } \\
\text { healthy donors before } \\
\text { cultivation } n=10\end{array}$} & \multicolumn{4}{|c|}{ Cultivation for 16 hours } \\
\hline & & $\begin{array}{l}\text { Lymphocytes of healthy } \\
\text { donors without extracts } \\
\qquad n=10\end{array}$ & $\begin{array}{c}\text { Lymphocytes of } \\
\text { donors + "Taban-Arshan" } \\
n=10\end{array}$ & $\begin{array}{c}\text { Lymphocytes of } \\
\text { donors + "Polyphytok-hol" } \\
n=10\end{array}$ & $\begin{array}{c}\text { Lymphocytes of } \\
\text { donors + "Baikalsky" } \\
n=10\end{array}$ \\
\hline CD3 & $68.44 \pm 2.7$ & $54.93 \pm 2.2$ & $57.78 \pm 2.1$ & $62.30 \pm 2.2 *$ & $55.60 \pm 3.1$ \\
\hline CD8 & $25.85 \pm 1.6$ & $28.47 \pm 1.2$ & $26.19 \pm 0.8$ & $29.75 \pm 2.1$ & $32.50 \pm 1.7^{*}$ \\
\hline CD16 & $10.82 \pm 0.5$ & $7.47 \pm 0.3$ & $8.20 \pm 1.1$ & $10.80 \pm 1.1$ & $12.90 \pm 1.1^{*}$ \\
\hline CD56 & $6.95 \pm 2.4$ & $7.78 \pm 2.0$ & $7.32 \pm 2.5$ & $2.40 \pm 2.5^{*}$ & $7.00 \pm 2.5$ \\
\hline
\end{tabular}

$* p<0.05$ (compared with lymphocytes of the healthy donors with no extracts used).

Table 2. Change of the expression of surface antigens of subpopulations of B-lymphocytes taken from the healthy donors during cultivation under the influence of the "Taban-Arshan", "Polyphytokhol" and "Baikalsky" extracts (in \%).

\begin{tabular}{|c|c|c|c|c|c|}
\hline \multirow[b]{2}{*}{$\begin{array}{l}\text { Lymphocyte } \\
\text { markers }\end{array}$} & \multirow{2}{*}{$\begin{array}{l}\text { Lymphocytes of } \\
\text { healthy donors before } \\
\text { cultivation } n=10\end{array}$} & \multicolumn{4}{|c|}{ Cultivation for 16 hours } \\
\hline & & $\begin{array}{l}\text { Lymphocytes of healthy } \\
\text { donors without extracts } \\
\qquad n=10\end{array}$ & $\begin{array}{c}\begin{array}{c}\text { Lymphocytes of } \\
\text { donors + "Taban-Arshan" } \\
n=10\end{array}\end{array}$ & $\begin{array}{c}\begin{array}{c}\text { Lymphocytes of } \\
\text { donors + "Polyphytok-hol" } \\
n=10\end{array}\end{array}$ & $\begin{array}{c}\text { Lymphocytes of } \\
\text { donors + "Baikalsky" } \\
n=10\end{array}$ \\
\hline $\mathrm{CD} 20$ & $10.77 \pm 0.5$ & $12.18 \pm 0.4$ & $10.20 \pm 0.6$ & $7.90 \pm 0.6^{*}$ & $7.20 \pm 0.63^{*}$ \\
\hline $\mathrm{CD} 72$ & $10.92 \pm 0.7$ & $9.70 \pm 0.6$ & $11.32 \pm 2.2$ & $7.10 \pm 2.1$ & $9.60 \pm 1.21$ \\
\hline $\mathrm{CD} 38$ & $7.40 \pm 2.1$ & $6.28 \pm 1.2$ & $5.78 \pm 0.7$ & $7.20 \pm 0.7$ & $2.80 \pm 0.73 *$ \\
\hline mIgM & $7.25 \pm 0.5$ & $6.72 \pm 0.9$ & $7.20 \pm 1.2$ & $5.10 \pm 1.2$ & $6.70 \pm 1.23$ \\
\hline $\mathrm{mIgG}$ & $6.53 \pm 0.6$ & $6.32 \pm 0.8$ & $6.90 \pm 1.9$ & $5.10 \pm 1.9$ & $3.70 \pm 1.94$ \\
\hline$\lambda$-chain Ig & $6.75 \pm 0.9$ & $6.68 \pm 0.8$ & $5.95 \pm 1.0$ & $6.00 \pm 0.8$ & $4.30 \pm 1.26$ \\
\hline$\kappa$-chain Ig & $12.48 \pm 2.9$ & $9.38 \pm 1.6$ & $8.35 \pm 1.9$ & $9.00 \pm 1.2$ & $8.20 \pm 1.06$ \\
\hline
\end{tabular}

$* p<0.05$ (compared with lymphocytes of the healthy donors with no extracts used).

Table 3. Change of the expression of activation antigens of lymphocytes taken from the healthy donors during cultivation under the influence of the "Taban-Arshan", "Polyphytokhol" and "Baikalsky" extracts (in \%).

\begin{tabular}{|c|c|c|c|c|c|}
\hline \multirow{2}{*}{$\begin{array}{l}\text { Activation } \\
\text { markers of } \\
\text { lymphocytes }\end{array}$} & \multirow{2}{*}{$\begin{array}{l}\text { Lymphocytes of } \\
\text { healthy donors before } \\
\text { cultivation } n=10\end{array}$} & \multicolumn{4}{|c|}{ Cultivation for 16 hours } \\
\hline & & $\begin{array}{l}\text { Lymphocytes of healthy } \\
\text { donors with-out extracts } \\
n=10\end{array}$ & $\begin{array}{c}\text { Lymphocytes of } \\
\text { donors + "Taban-Arshan" } \\
n=10\end{array}$ & $\begin{array}{c}\text { Lymphocytes of } \\
\text { donors + "Polyphytok-hol" } \\
n=10\end{array}$ & $\begin{array}{c}\text { Lymphocytes of } \\
\text { donors + "Baikalsky" } \\
n=10\end{array}$ \\
\hline CD23 & $5.73 \pm 0.40$ & $4.90 \pm 0.01$ & $6.80 \pm 2.10$ & $6.05 \pm 2.85$ & $8.25 \pm 3.75$ \\
\hline CD25 & $5.88 \pm 0.47$ & $5.18 \pm 0.29$ & $5.40 \pm 0.42$ & $5.00 \pm 0.42$ & $6.30 \pm 0.42$ \\
\hline CD71 & $7.60 \pm 1.43$ & $8.20 \pm 1.38$ & $7.34 \pm 0.71$ & $5.70 \pm 0.71$ & $10.60 \pm 0.71$ \\
\hline HLA-DR & $12.15 \pm 2.9$ & $10.78 \pm 2.27$ & $10.40 \pm 2.34$ & $5.60 \pm 2.3^{*}$ & $4.00 \pm 2.34^{*}$ \\
\hline CD95 & $6.57 \pm 1.68$ & $7.64 \pm 1.44$ & $7.05 \pm 1.06$ & $6.20 \pm 1.06$ & $6.40 \pm 1.06$ \\
\hline CD54 & $5.72 \pm 0.79$ & $4.91 \pm 0.65$ & $5.10 \pm 0.75$ & $5.50 \pm 0.50$ & $7.80 \pm 0.20$ \\
\hline
\end{tabular}

$* p<0.05$ (compared with lymphocytes of the healthy donors with no extracts used). 
investigation has also demonstrated immunosuppressive effects of the "Polyphytokhol" and "Baikalsky" extracts on the processes of lymphocytes differentiation. The conducted studies make it possible to conclude that the "Taban-Arshan" extract of the Tibetan medicine is the only one of the tested drugs, which has neither activating nor inhibiting effects on the expression of the surface receptors of the healthy donors' lymphocytes.

At the next step of the research effects of the "Taban-Arshan","Polyphytokhol" and "Baikalsky" extracts on the lymphocytes taken from the patients with early rheumatoid arthritis were investigated.

Previous studies have shown that a reduction of T-lymphocytes in the blood due to helper cells and a decrease of quantity of mature B-lymphocytes primed by an antigen are typical of the early rheumatoid arthritis. An increase of lymphocyte expression CD54 receptor of cell adhesion and CD23, CD25, CD71, HLA-DR, CD95 activation markers was also noticed [18-25].

During the cultivation of the lymphocytes taken from the patients with early rheumatoid arthritis no changes were registered related to normalization or improvement of any disturbed parameters of the surface lymphocyte markers when the "Polyphytokhol" and "Baikalsky" extracts were used. The "Polyphytokhol" extract raised the $\mathrm{mIgG}, \mathrm{CD} 71$, HLA-DR expression, and the "Baikalsky" extract considerably increased the quantity of CD8, CD56, which had already been high in the cell culture of the patients with early rheumatoid arthritis. It was also noticed that due to the extract effect, the number of CD4-lymphocytes was decreased though their level was already low in such patients. One should pay attention to the fact that the "Polyphytokhol" and "Baikalsky" extracts increase ICAM-1 expression, which indicates advisability of their use as anti-inflammatory drugs of folk medicine. However, taking into account their negative effects on the immune system, it is not expedient to use them as immunomodulators in case of autoimmune diseases.

The results of the analysis of the "Taban-Arshan" extract's effect on the lymphocytes picked out of the patients with early RA show that it does not affect the expression of T-lymphocytes and CD16 and CD56 markers. If the extract is added to the lymphocytes of patients with early RA, during the cultivation there appears a tendency to lowering the expression of the marker of B-lymphocytes CD20. It indicates a decrease of potential readiness for B-cells activation. In this connection an analysis of changes in the content of B-lymphocytes of various degree of differentiation patients with the early RA during the cultivation process was performed. The "Taban-Arshan" extract affects the content of differentiated forms of the B-lymphocytes obtained from the ex- amined patients. A valid reduction of the number of plasma cells $(\mathrm{CD} 38) p<0.05$ under influence of the extract (Figure 1) compared with the similar index for the healthy one is regular.

A low level of expression of both receptors of CD38 and $\mathrm{mIgM}^{+}-, \mathrm{mIgG}^{+}$-lymphocytes confirms the fact that mature B-cells affected by the extract are neither activated nor transformed into antibody producing cells.

The examined extract exerts a marked inhibitory action on the activated B-lymphocytes that express the FcRII (CD23 ${ }^{+}$-cells) receptor; in the "Taban-Arshan" extract presence (Figure 2), this index goes down from $14.49 \% \pm 1.16 \%$ to $8.21 \% \pm 0.54 \%$. It means that there is deceleration of activation processes within the B-cell part of the immune system.

The effect of the examined phytopreparation results in a tendency of lowering the expression of the receptor for interleukin-2 (CD25), the receptor for transferrin (CD71) and the expression of the late activation antigen (HLA-DR ${ }^{+}$-lymphocytes), that reflects processes of differentiation of lymphocytes, the expression of which approached similar values of the healthy donors. The increase of the lymphocytes expressing the adhesion ICAM (CD54) in the cell cultures of the examined patients show that in case of early rheumatoid arthritis the

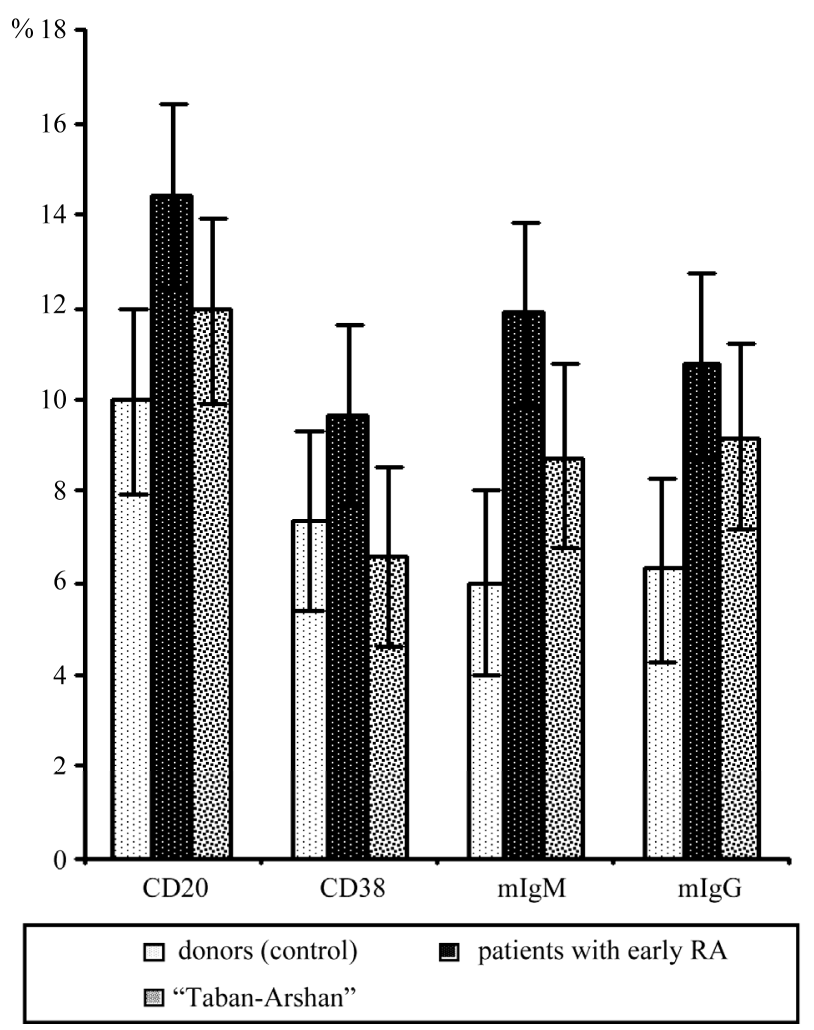

Figure 1. Expression of the surface antigens of the B-lymphocyte subpopulation in the patients with early rheumtoid arthritis with the "Taban-Arshan" extract used. 


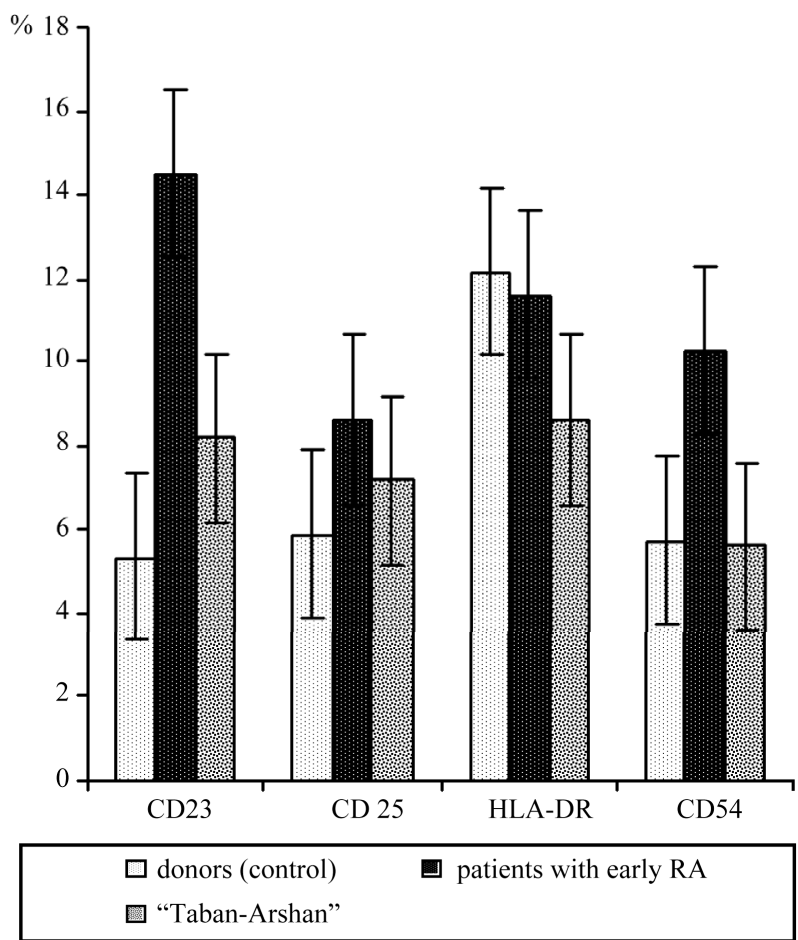

Figure 2. Expression of activation antigens by lymphocytes in the patients with early rheumatoid arthritis with the "TabanArshan" extract used.

processes of the immune system stimulation are not switched off. [26-28]. The "Taban-Arshan" extract causes a valid reduction in the expression of this marker to the level of the healthy donors (Figure 2).

The increase of the expression of membrane CD30 during an autoimmune inflammation is based on a mechanism of involving the inflammatory cells in an antigen response reaction. So the patients with early rheumatoid arthritis have an increase of the CD30 surface receptor from $0.73 \% \pm 0.76 \%$ up to $3.68 \% \pm 0.60 \%$ registered for the donors $[29,30]$, however in the presence of the extract this figure decreases to $2.90 \% \pm$ $0.25 \%$.

The results of this experimental section prove that, on the one hand, there is no significant effect of the examined extract on expression of either T-lymphocyte markers or NK-cells (CD16) and activated NK-cells (CD56) in the patients with early rheumatoid arthritis.

On the other hand, it has been noticed that the "Taban-Arshan" extract exerts a slight inhibitory effect on the expression of early activation antigens of lymphocytes CD25, CD71, as well as of late activation antigen HLA-DR and surface immunoglobulins $\mathrm{M}$ and $\mathrm{G}$.

It has been determined that the "Taban-Arshan" extract reduces the expression of molecules of cell-cell adhesion ICAM-1, expression of low-affinity receptor for IgE on activated B-lymphocytes, the quantity of plasma cells
CD38 and expression of surface receptor CD30.

Thus, the "Taban-Arshan" extract of the Tibetan medicine produces an immunomodulatory effect: it inhibits apparent activation of the B-cell part of the immune system in patients with early rheumatoid arthritis. The revealed immunomodulatory effect suggests that the "Taban-Arshan" extract can be used for patients with other autoimmune diseases, which have similar immune status disorders.

The conducted comparative studies of the Tibetan medicine extracts make it possible to single out the "Taban-Arshan" extract as having the apparent immunomodulatory effect. Our studies have proved the "Taban-Arshan" extract ability to correct an immune response in inflammatory diseases: the "Taban-Arshan" extract corrects the changes of the immune system characterized by evident activation of the B-cell part of the immune system and normalizes immune parameters of the lymphocytes taken from the patients with autoimmune diseases (early rheumatoid arthritis). The results of our studies confirm that the immunocorrective effect of the "Taban-Arshan" extract is connected with its ability to suppress increased activation of lymphocytes by normalizing expression of the main activation antigens (CD23, CD25, CD71, HLA-DR, CD54).

Among the three examined preparations, the "Taban-Arshan" extract proves to be most effective for correction of immune disturbances in early rheumatoid arthritis. Evidently, the immunomodulatory function of the extract during the cell cultivation process, which we have revealed, can produce similar actions on activated lymphocytes in the human organism.

\section{REFERENCES}

[1] Proskuryakov, S.Y. and Gabai, VL. (2010) Mechanisms of tumor cell necrosis. Current Medicinal Chemistry, 16, 2010, 56-68. doi:10.2174/138161210789941793

[2] Flad, H.D. and Brandt, E. (2010) Platelet-derived chemokines: Pathophysiology and therapeutic aspects. Cellular and Molecular Life Sciences, 7, 2010.

[3] Stromberg, S.P. and Carlson, J.M. (2010) The suppression of immune system disorders by passive attrition. PLoS One, 5, 2010, 9648-9649.

[4] Bach, J.F. (2005) Infections and autoimmune diseases. Journal of Autoimmunity, 25, 2005, 74-80. doi:10.1016/j.jaut.2005.09.024

[5] Liu, Y., Dao, Z., Yang, C., Liu, Y. and Long, C. (2009) Medicinal plants used by Tibetans in Shangri-la, Yunnan, China. Journal of Ethnobiology and Ethnomedicine, 5, 2009, 15-16. doi:10.1186/1746-4269-5-15

[6] Zhang, Z., Luo, P., Li, J., Yi, T., Wang, J., An, J. and Zhang, H. (2008) Comparison of the antiinflammatory activities of three medicinal plants known as "meiduoluomi" in Tibetan folk medicine. Yakugaku Zasshi, 128, 2008, 805-810. doi:10.1248/yakushi.128.805

[7] Darshan, S. and Doreswamy, R. (2004) Patented antiin- 
flammatory plant drug development from traditional medicine. Phytotherapy Research, 18, 2004, 343-357. doi:10.1002/ptr.1475

[8] Talhouk, R.S., Karam, C., Fostok, S., El-Jouni, W. and Barbour, E.K. (2007) Anti-inflammatory bioactivities in plant extracts. Journal of Medicinal Food, 10, 2007, 1-10.

[9] Olivo, E.L. (2009) Protection throughout the life span: The psychoneuroimmunologic impact of Indo-Tibetan meditative and yogic practices. The New York Academy of Sciences, 1172, 2009, 163-171. doi:10.1111/j.1749-6632.2009.04415.x

[10] Badmaev, P.A. (1991) Fungamentals of the Tibetan medical science Chzhud-shi. Science.

[11] Ayusheeva, L.V. (2006) Tibetan medicine in Buryatiya. Problems of Social Hygiene, Health Protection and History of Medicine, 5, 58-60.

[12] Serebrennikova, Yu.A., Sakanyan, E.I., Kabyshev, K.E. and Sakanyan, K.M. (2005) Balneotherapy in today medical practice. Herald of VTU Series: Chemistry, Biology and Pharmacy, 1, 225-235.

[13] Semenova, L.S., Sakanyan, E.I., Blinova, K.F., Bal'zhirov, B.G., Sambueva, Z.G., Merdygeeva, A.K., Moldaver, B.L. and Bartanov, A.I. (1997) "Taban-Arshan" phytopreparation for a balneal practice. Tibetan medicine. Traditions, perspectives, integration: Theses of reports on scientific-practice conference. Ulan-Ude, 80-82.

[14] Korsun, V.F., Nikolarv, S.M., Dargaeva, T.D., Rossiyskaya, G.I., Korsun, E.V. and Aleksandrov, Yu.N. (2004) Polyphytochol in therapy of liver diseases. Practical Phytotherapy, 3, 43-47.

[15] Dashinamzhilov, Zh.B. (2008) Therapeutic effectiveness of "Polyphytochol" phytocollection in a complex therapy of alcohol damages of liver of patients with chronic alcoholism. Narcology Issues: Science and Practice, 4, 90-96.

[16] Butov, M., Korsun, V., Korsun, E. and Aleksandrov, Yu. (2005) Polyphytochol at functional disfunction of the biliary tract. Doctor, 1, 60-61.

[17] Ubeeva, I.P., Ayusheva, S.Ts., Baldanov, B.V. and Zherbanova, L.V.(2007) An effect of "Baikalsky-7" phytocollection on chronic toxic hepatitis. Bulletin of the Eastern Siberian Scientific Center, 5, 54-56.

[18] Potanin, A.Y., Timofeev, V.T., Shostak, N.A. and Anichkov, D.A. (2006) Phenotype of peripheral blood lymphocytes and serum immunoglobulin concentration in patients with early rheumatoid arthritis. Bulletin of Experimental Biology and Medicine, 141, 2006, 84-86.

[19] Pincus, T. and Sokka, T. (2008) Mortality in rheumatic diseases: Introduction. Clinical and Experimental Rheumatology, 26, 35-61.

[20] Miranda-Carús, M.E., Balsa, A., Benito-Miguel, M., Pérez de Ayala, C. and Martín-Mola, E. (2004) IL-15 and the initiation of cell contact-dependent synovial fibroblast-T lymphocyte cross-talk in rheumatoid arthritis: Effect of methotrexate. The Journal of Immunology, 173,
2004, 1463-1476.

[21] Ancuța, C., Ancuța, E., Iordache, C., Ceauşu, M. and Chirieac, R. (2009) Immunohistochemical study of skeletal muscle in rheumatoid myositis. Romanian Journal of Morphology and Embryology, 50, 2009, 223-227.

[22] Shimojima, Y., Matsuda, M., Ishii, W., Gono, T. and Ikeda, S. (2008) Analysis of peripheral blood lymphocytes using flow cytometry in polymyalgia rheumatica, RS3PE and early rheumatoid arthritis. Clinical and Experimental Rheumatology, 26, 2008, 1079-1082.

[23] Ria, F., Penitente, R,, De Santis, M., Nicolò, C., Di Sante, G., Orsini, M., Arzani, D., Fattorossi, A., Battaglia, A. and Ferraccioli, G.F. (2008) Collagen-specific T-cell repertoire in blood and synovial fluid varies with disease activity in early rheumatoid arthritis. Arthritis Research \& Therapy, 10, 2008, R135. doi:10.1186/ar2553

[24] Haas, C., Aicher, W.K., Dinkel, A., Peter, H.H. and Eibel, H. (1997) Characterization of SV40T antigen immortalized human synovial fibroblasts: Maintained expression patterns of EGR-1, HLA-DR and some surface receptors. Rheumatology International, 16, 1997, 241-247. doi:10.1007/BF01375656

[25] Galligan, C.L., Siebert, J.C., Siminovitch, K.A., Keystone, E.C., Bykerk, V., Perez, O.D. and Fish, E.N. (2009) Multiparameter phospho-flow analysis of lymphocytes in early rheumatoid arthritis: Implications for diagnosis and monitoring drug therapy. PLoS One, 20, 2009, 6703.

[26] Klimiuk, P.A., Fiedorczyk, M., Sierakowski, S. and Chwiecko, J. (2007) Soluble cell adhesion molecules (sICAM-1, sVCAM-1, and sE-selectin) in patients with early rheumatoid arthritis. Scandinavian Journal of Rheumatology, 36, 2007, 345-350.

[27] Montecucco, F., Burger, F., Pelli, G., Poku, N.K., Berlier, C., Steffens, S. and Mach, F. (2009) Statins inhibit C-reactive protein-induced chemokine secretion, ICAM-1 upregulation and chemotaxis in adherent human monocytes. Rheumatology, 48, 233-242.

doi:10.1093/rheumatology/ken466

[28] Lee, S.I., Lee, S.Y., Yoon, K.H., Choi, K.S., Jang, K.Y., Yoo, W.H., Kim, S.H., Choi, T.H. and Park, J.G. (1999) Molecular MR imaging for visualizing ICAM-1 expression in the inflamed synovium of collagen-induced arthritic mice. Korean Journal of Radiology, 10, 1999, 472-480. doi:10.3348/kjr.2009.10.5.472

[29] Gerli, R., Bistoni, O., Lunardi, C., Giacomelli, R., Tomassini, C., Biagini, P. and Pitzalis, C. (1999) Soluble CD30 in early rheumatoid arthritis as a predictor of good response to second-line therapy. Rheumatology, 38, 1999, 1282-1284. doi:10.1093/rheumatology/38.12.1282

[30] Savolainen, E., Matinlauri, I., Kautiainen, H., Luosujärvi, R. and Kaipiainen-Seppänen, O. (2008) Serum soluble CD30 in early arthritis: A sign of inflammation but not a predictor of outcome. Clinical and Experimental Rheumatology, 26, 2008, 922-925. 